\title{
Editorial
}

\section{Foraging Biology of Neglected Bee Pollinators}

\author{
C. Rasmussen, ${ }^{1}$ J. Nieh, ${ }^{2}$ and J. C. Biesmeijer ${ }^{3}$ \\ ${ }^{1}$ Department of Biological Sciences, Aarhus Universitet, Ny Munkegade 120, 8000 Århus C, Denmark \\ ${ }^{2}$ Section of Ecology, Behavior, and Evolution, Division of Biological Sciences, University of California San Diego, \\ Mail Code 0116, 9500 Gilman Drive, La Jolla, CA 92093-0116, USA \\ ${ }^{3}$ Department of Biological Sciences, Earth and Biosphere Institute, University of Leeds, Leeds LS2 9JT, UK
}

Correspondence should be addressed to J. Nieh, jnieh@ucsd.edu

Received 12 April 2010; Accepted 12 April 2010

Copyright (C) 2010 C. Rasmussen et al. This is an open access article distributed under the Creative Commons Attribution License, which permits unrestricted use, distribution, and reproduction in any medium, provided the original work is properly cited.

Charles Darwin [1] was fascinated by bees, particularly bumble bees, and even recruited his children to trace their meandering paths through his garden [2]. Darwin's curiosity is part of a long history of interest in bee foraging biology. This attention is well deserved because bees are key pollinators in diverse ecosystems, exhibit complex adaptations such as recruitment communication, use foraging strategies that adapt to conditions inside and outside the nest, and have evolved elegant solutions to the challenges of gathering scattered floral resources. Much research has focused on honey bees, a remarkable model organism. However, the goal of this special issue is to explore new research on somewhat "neglected" bee pollinators. Many of these bees are not traditional model systems but have recently received attention because they are native pollinators whose diversity and numbers are in decline [3]. In addition, concern about the decline of honey bees (Apis mellifera), a frequently used agricultural pollinator, has led to increasing public awareness that non-Apis species can also pollinate crops. This has increased interest in alternative pollinators, many of which are native bee species.

This special issue therefore examines a wide range of bee species and is divided into three sections: (1) bee floral preferences in mixed landscapes, (2) the agricultural role of bees, and (3) influences on bee foraging activity. The first section, floral preferences, provides data on the abundance of different species and what they feed on. We then examine the role of diverse bee species in pollinating agricultural crops. Finally, we explore different factors that influence bee foraging activity inside and outside the nest.
Floral Preferences (C. Rasmussen). Single bee species never visit all of the different flowers in an area. Constraints on floral morphology or flowering phenology may prevent them from doing so, but even more interesting, most bees have a preference for pollen of certain plant species. This section traces the preferred floral resources for three different groups of bees: Ceratina, Halictus, and Bombus. Kobayashi-Kidokoro and Higashi examined pollen loads brought back to the nest by the small carpenter bee (Ceratina flavipes) and found that, within a bee population, such loads consist of 14 different pollen sources, although a single bee rarely exploits more than three different plants for pollen. While such preferences could be guided by local floral abundance, the authors found that preference for certain pollen sources persisted, even when the plant was uncommon. This phenomenon is termed flower constancy, where individual pollinators prefer flowers of the same species that they are already foraging at, thus bypassing other available flower species, even if the other flowers may be more rewarding. Polidori and collaborators likewise report a limited range of pollen sources for Lasioglossum malachurum, which can use from five to seven different pollen types but only visit one to two different plant species during each foraging flight. This species, however, exhibits large annual variation in the preferred pollen type. Lastly, Irene Konovalova compiled all known information about the bumblebees (Bombus) of Ukraine. Most of these bumblebees are polylectic with regional and seasonal preferences to the same flowering plant species, with the exception of $B$. gerstaeckeri, which almost exclusively forages from Aconitum. 
Bees and Agriculture (J. Nieh). This section begins with papers examining the role of two solitary bee species in crop pollination. Güler and Sorkun report that Andrena flavipes (Andrenidae) is not an important pollinator of sweet cherry but does collect pollen from a wide variety of plants, particularly the Brassicaceae. Matsumoto and Maejima examine apple pollination by the megachilid bee, Osmia cornifrons. Interestingly, males and females of this solitary species collect nectar and, in net enclosure experiments, males contribute to apple pollination. Next, Keasar reviews the agricultural role of carpenter bees, which exhibit a wide range of solitary to quasisocial or communal organization. He describes the benefits and difficulties of using these bees as agricultural pollinators. Rao and Stephen then examine a thriving population of native bumble bees in western Oregon (USA) and suggest that cultivation of different crops blooming in succession may account for the diversity and strength of this population. Finally, Brunet and Steward study a guild of bee pollinators and report that some native bee species are more effective than honey bees at alfalfa pollination. Together, these studies highlight the agricultural importance of native bee species and suggest directions for future research, such as the role of solitary bee male pollinators and the need to determine the relative pollinating efficiencies of diverse bee species.

Influences on Foraging Activity (J. C. Biesmeijer). The "neglected pollinators" we are reporting on in this issue are primarily flower-visitors in search of food. Pollination is a side-effect of their foraging activities. The individual insects have to make decisions on when to forage, where to forage, and what to collect. The contributions in this section shed some more light on each of these three areas. Most bees have the difficult task of optimizing foraging and guarding their offspring in the nest. Lienhardt and collaborators show that, in the primitively eusocial bee Halictus scabiosae, the start of foraging in the morning depends on air temperature. Unexpectedly, however, they found that foraging ceases in the afternoon under excellent weather conditions, and bees even close the nest entrances. This is probably a response to the risk of nest parasitism by cleptoparasitic bees, whose activity patterns are largely asynchronous to that of the hosts. Eusocial bees do not have this optimization problem, because there are always some workers that remain in the colony and can defend the nest. In that case one would expect a more direct link between temperature and foraging activity. Couvillon et al. show that this is not the case for small and large bumblebee workers of the same colony. One might have expected larger bees to start foraging at lower temperatures than small worker and to cease foraging earlier at high temperatures, but this is clearly not the case for the North-American bumblebee, Bombus impatiens. Once foraging has started and flowers have been found, decisions have to be made on where to forage and how long to stay at flowers. Taneyhill presents a new more general model aiming to explain the decision of departure from a flower and tests the new model and a previous threshold model empirically. In addition to external factors, there are internal factors influencing foraging activity, particularly in social bees. Nunes-Silva and colleagues report on a Brazilian stingless bee, Plebeia remota, where worker production stops during the colder winter months. Temperature and relative humidity affected foraging activity as expected. However, in addition they found that when brood production stopped, bees mainly collected nectar and rarely pollen. This has been reported before in honeybees but was not known from these tropical stingless bees.

\section{Rasmussen J. Nieh \\ J. C. Biesmeijer}

\section{References}

[1] C. R. Darwin, On the Origin of Species, John Murray, London, UK, 1859.

[2] R. B. Freeman, "Charles Darwin on the routes of male humble bees," Bulletin of the British Museum (Natural History) Historical Series, vol. 3, pp. 179-189, 1968.

[3] J. C. Biesmeijer, S. P. M. Roberts, M. Reemer et al., "Parallel declines in pollinators and insect-pollinated plants in Britain and the Netherlands," Science, vol. 313, no. 5785, pp. 351-354, 2006. 

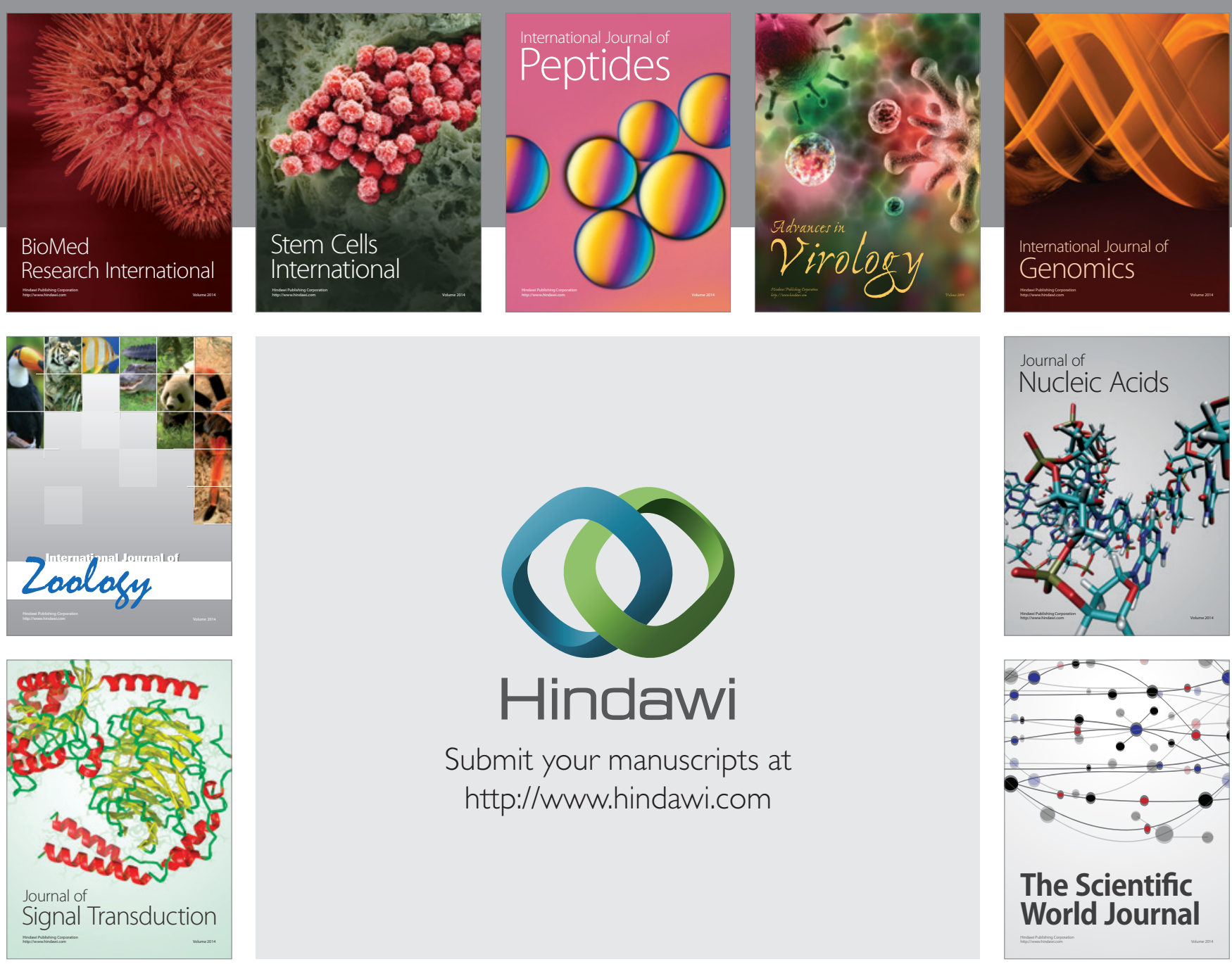

Submit your manuscripts at

http://www.hindawi.com
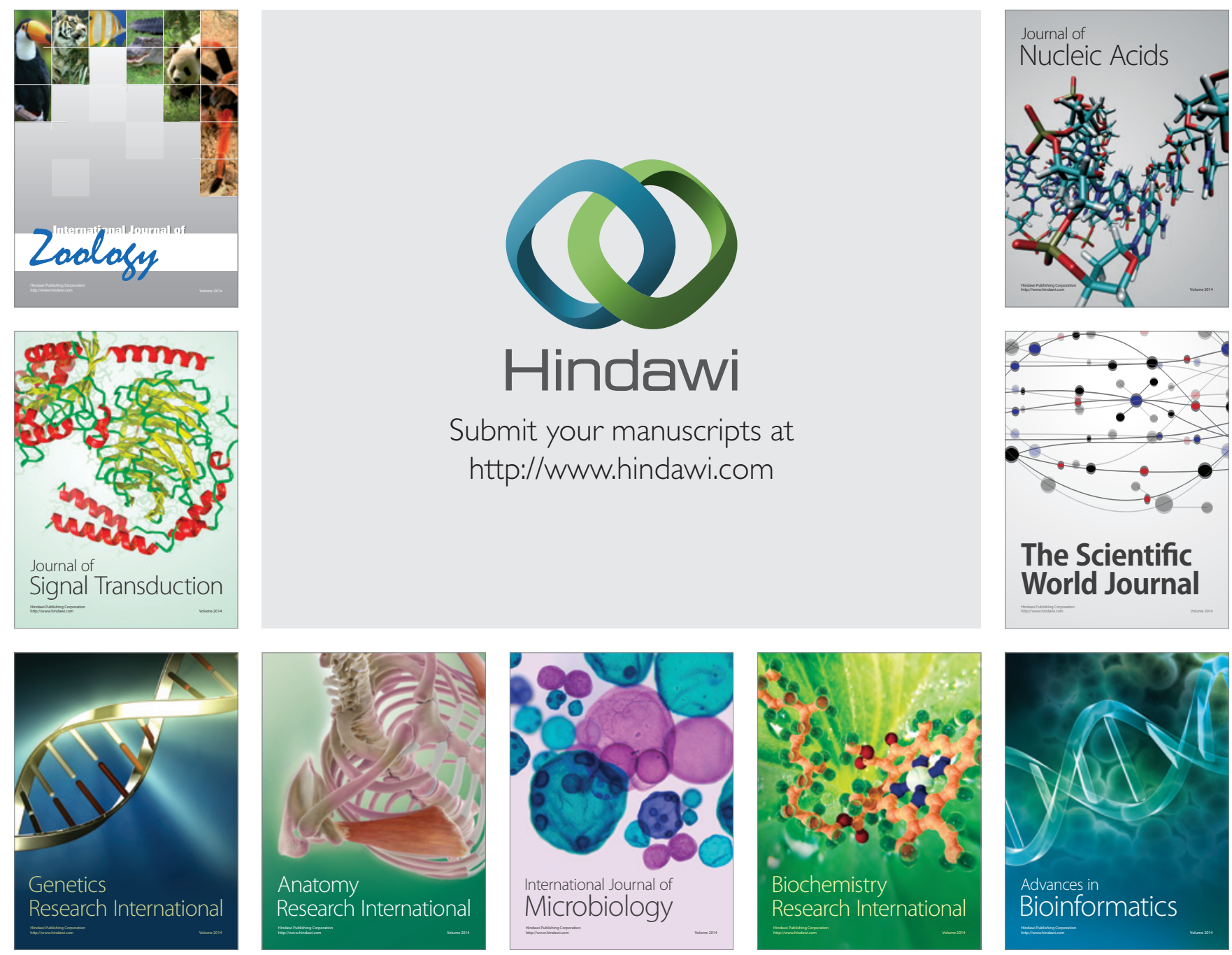

The Scientific World Journal
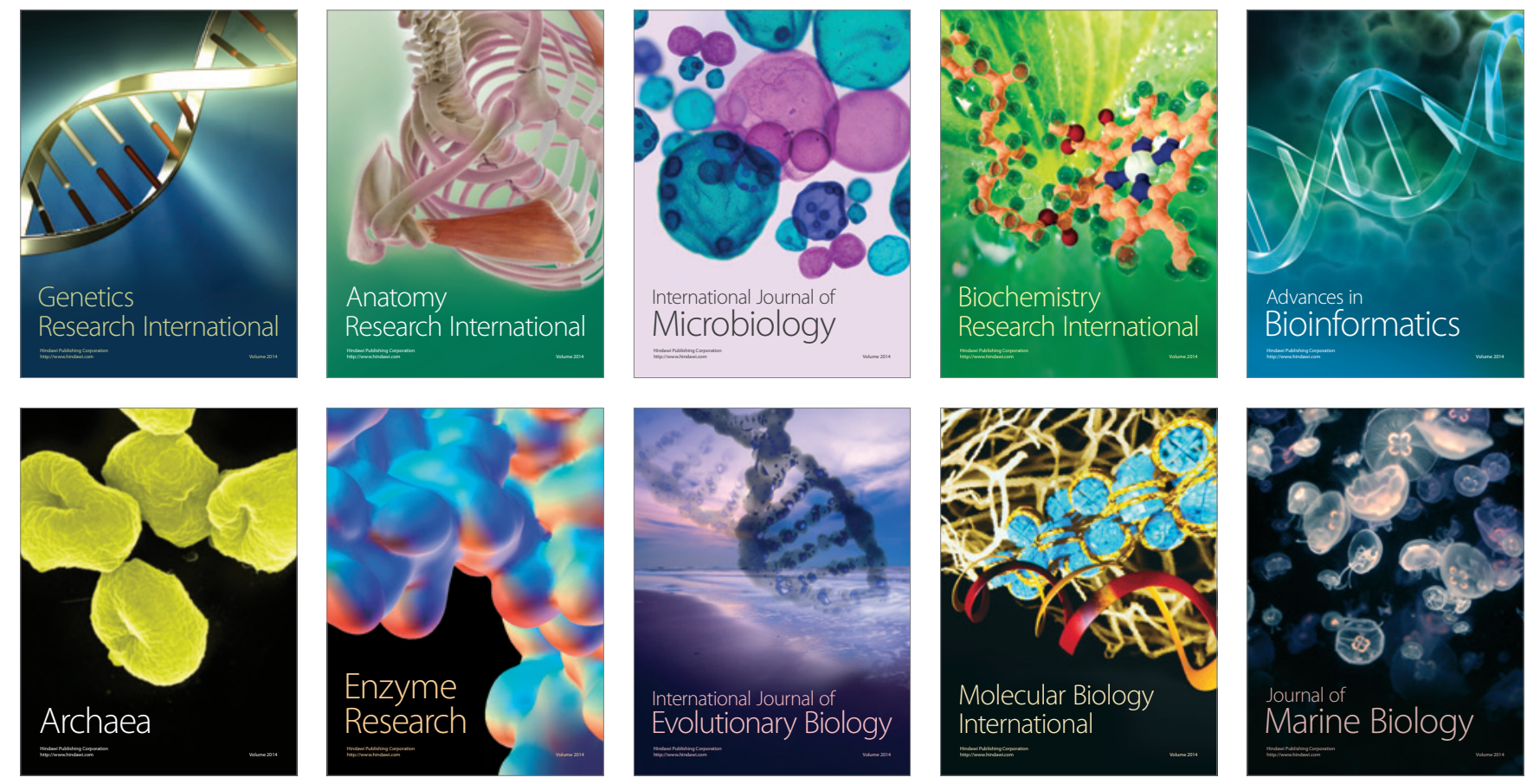